\title{
May Neutrophil to Lymphocyte Ratio Serve a Role in the Prediction of Clinical Features of Migraine?
}

\author{
Halil Onder ${ }^{\mathrm{a}, \mathrm{c}}$, Muhammed Mustafa Deliktas ${ }^{\mathrm{b}}$
}

\begin{abstract}
Background: We aimed to investigate the possible association between the value of neutrophil to lymphocyte ratio (NLR) and some specific features of migraine.

Methods: We have included initially all the patients with migraine who applied to our neurology clinic in Yozgat City Hospital during December 2019 and agreed to participate in this study. The demographic and clinical characteristics including migraine subtype (episodic/chronic), headache frequency per month, headache characteristics of all patients were interrogated. Besides, the presence of fibromyalgia (FM) and chronic fatigue syndrome (CFS) was also noted. The severity of migraine was assessed using the headache impact test (HIT-6) and the severity of chronic fatigue was assessed using the Functional Assessment of Chronic Illness Therapy (FACIT) fatigue scale. A hemogram was performed upon admission to the clinic. The patients with migraine attacks during the clinic visits were excluded from the study.
\end{abstract}

Results: Ultimately, 52 migraineurs were included in this study. The mean age was $37.34 \pm 11.80$ and the female/male $(\mathrm{F} / \mathrm{M})$ ratio was $49 / 3$. Thirty-six patients $(69 \%)$ were diagnosed with episodic migraine (EM), and 16 of them (31\%) were diagnosed with chronic migraine (CM). The results of the comparative analyses between EM and CM groups showed that the NLR did not differ between groups. To evaluate the possible association of the NLR with other clinical parameters, additional comparative analyses evaluating the presence of FM, CFS, cognitive symptoms and tinnitus were also conducted, which showed no significant differences. The results of the correlation analyses to evaluate the possible associations between the NLR value and other clinical parameters were also unremarkable.

Conclusions: We suggest that the NLR cannot be a specific marker to be used in the differential diagnosis or prediction of any features of migraines during the interictal period. However, in light of the previous reports, the utility of this value in the differentiating of migraine attacks from other causes of headache attacks can be investigated in

Manuscript submitted February 17, 2020, accepted March 2, 2020

aDepartment of Neurology, Yozgat City Hospital, Yozgat, Turkey bepartment of Psychology, Yozgat City Hospital, Yozgat, Turkey ${ }^{\mathrm{c} C}$ Corresponding Author: Halil Onder, Neurology Clinic, Yozgat City Hospital, Yozgat, Turkey. Email: halilnder@yahoo.com

doi: https://doi.org/10.14740/jnr574 future related studies.

Keywords: Migraine; Inflammation; Neutrophil to lymphocyte ratio; Marker

\section{Introduction}

Migraine may be described as a neurovascular disorder, of which neurogenic inflammation, contractile dysfunction of cranial blood vessels, and depression mechanisms extending from the cerebral cortex constitute the critical pathophysiological mechanisms $[1,2]$. Neuroinflammatory conditions, cytokines, various neuropeptides, and vasomotor changes have been hypothesized to be responsible for the pathogenesis of migraine headaches [3]. Various hematological markers may be used for the evaluation of the inflammatory processes. The white blood cell (WBC) count is one such marker, used widely in clinical practice and easily measured, reproducible, and inexpensive. However, even when WBC is within normal limits, an increased neutrophil to lymphocyte ratio (NLR) may indicate inflammation and inflammation-related pathologies [4]. The NLR has been used in the evaluation and prognostication of various diseases, including coronary artery diseases, malignancies, rheumatologic diseases, and neurological diseases [512]. Few studies have investigated the value of this marker in the evaluation of migraineurs $[8,10,13]$. These studies found that NLR in migraineurs during the attack period to be higher than that in healthy controls. However, the association of this value with the clinical features of migraineurs during the interictal period has not been studied previously in a systematic and detailed manner. This study aims to investigate the possible association of the NLR during interictal period of migraine with some specific features of the disease.

\section{Materials and Methods}

\section{Study population}

All patients with migraine who applied to the neurology clinic in Yozgat City Hospital during December 2019 and agreed to participate in this study were initially included in the study. The diagnosis of migraine headaches in the patients was based on the four diagnostic criteria of the International Classification 
of Headache Disorders third edition [14]. The patients were questioned regarding their demographic and clinical characteristics, including migraine subtype (episodic/chronic), headache frequency per month, and headache characteristics. Based on previous reports emphasizing the frequent coexistence of either fibromyalgia (FM) and chronic fatigue syndrome (CFS) with migraine and the possibility of common pathophysiological mechanisms among these disorders, the presence of FM and CFS was also noted. The diagnosis of FM was made according to ACTTION-APS Pain Taxonomy diagnostic criteria [15], and the diagnosis of CFS was made based on the National Institute for Health and Care Excellence guidelines [16]. The presence of symptoms of cognitive disturbances was also noted. The severity of migraine was assessed using the headache impact test (HIT-6), and the severity of chronic fatigue was assessed using the Functional Assessment of Chronic Illness Therapy (FACIT) fatigue scale. A hemogram was performed upon admission to the clinic. The patients who suffered migraine attacks during the clinic visit were excluded from the study. Patients with an infectious disease (urinary tract infection, sinusitis, cellulitis, or otitis media), malignancy, heart failure, coronary artery disease, pulmonary disease, or chronic inflammatory disease were also excluded from the study, as these may have affected the results. Written informed consent was obtained from all patients.

\section{Instruments}

\section{HIT-6}

The HIT-6 [17] is a validated tool to assess the impact of headache (range: 36 - 78) on the lives of respondents. HIT-6 scores $\leq 48$ reflect little impact; scores between 56 and 59 reflect a substantial impact, and scores $\geq 60$ reflect severe impact.

\section{FACIT fatigue scale}

The FACIT fatigue scale is a short, 13-item, easy to administer tool that measures an individual's level of fatigue during their usual daily activities over the past week. The FACIT fatigue scale is easy to complete (in $5-10 \mathrm{~min}$ ) and is written at the fourth-grade reading level. It has demonstrated equivalence in the mode of administration (interview vs. self-report) and can be used in a variety of clinical settings. The validity and reliability study has been carried out by Chandran et al [18].

\section{Laboratory analysis}

All values in the study were calculated from patients' complete blood count (CBC) analysis, which was investigated at the time of admission to the polyclinic. NLRs were calculated for all patients from their $\mathrm{CBC}$ results as dividing the number of neutrophils by the number of lymphocytes. Routine electronic CBC device was used (Cell-Dyne 3700, Abbott, Abbott Park, IL, USA) for this purpose.

\section{Statistical analyses}

All statistical analyses were performed using the SPSS statistics 20 programs. Continuous variables are expressed as mean \pm standard deviation and median (range) according to the type of the data being parametric or non-parametric. The compliance of the variables with normal distribution was assessed by the Shapiro-Wilk tests. Inter-group analyses were performed with Student's $t$-test for normally distributed variables and the Mann-Whitney U test for non-parametric variables. The Chisquare test was used for the comparison of qualitative data. To determine the correlation between the variables, Spearman's rank or Pearson's correlation analyses were performed according to the distribution of the data. The number of independent factors was determined according to the study sample size. A $P$ value of $<0.05$ was considered to be statistically significant.

This study was approved by the institutional review board, and was conducted in accordance with the declaration of Helsinki.

\section{Results}

Ultimately, 52 migraineurs were included in this study. The mean age was $37.34 \pm 11.80$, and the female/male ratio was $49 / 3$. Thirty-six patients $(69 \%)$ were diagnosed with episodic migraine (EM), and 16 of them (31\%) were diagnosed with chronic migraine (CM). The mean body mass index (BMI) of the group was $27.5 \pm 5.4$. FM was present in 26 of the patients $(50 \%)$; CFS was present in 47 of them $(90 \%)$. Other clinical features of the group are presented in Table 1.

The results of comparative analyses between the EM and CM groups showed that the NLR did not differ between the groups. The EM and CM groups were similar in terms of age, gender, and BMI. While FM and CFS were more common in the CM group, this did not reach statistical significance. The score on the FACIT fatigue scale was higher in the CM group; although also not statistically significant, it was close to significance $(\mathrm{P}=0.056)$. The results of the comparative analyses are presented in detail in Table 2.

To evaluate the possible association of the NLR with other clinical parameters, additional comparative analyses were conducted. No significant differences were found in the NLR value associated with the presence of FM, CFS, cognitive symptoms, or tinnitus (Table 3).

Further correlation analyses were conducted to evaluate possible associations between the NLR and other clinical parameters. The correlations that reached significance were between the FACIT fatigue scale score and HIT-6 $(r=0.322$, $\mathrm{P}=0.020)$, and between the FACIT fatigue scale score and headache frequency per month $(\mathrm{r}=0.497, \mathrm{P}=0.000)$ (Table 4).

\section{Discussion}

The present study found no difference in the NLR between the EM and CM groups. Furthermore, no significant association was found between the NLR and any of the clinical parameters 
Table 1. The Demographic and Clinical Features of the Overall Patient Group

\begin{tabular}{ll}
\hline & Overall patient group $(\mathbf{n}=\mathbf{5 2})$ \\
\hline Age (mean \pm SD) & $37.3 \pm 11.8$ \\
Gender (F/M) & $49 / 3$ \\
Episodic/chronic & $36 / 16$ \\
BMI (mean \pm SD) & $27.5 \pm 5.4$ \\
Tinnitus & $26 / 52$ \\
Fibromyalgia & $26 / 52$ \\
Chronic fatigue syndrome & $47 / 52$ \\
The presence of cognitive symptoms & $40 / 52$ \\
FACIT fatigue scale (mean \pm SD) & $30.5 \pm 10.1$ \\
Headache impact test (mean \pm SD) & $23.0 \pm 3.7$ \\
Headache frequency per month (median (range)) & $8(23)$ \\
NLR (median (range)) & $2.0(7.8)$ \\
\hline
\end{tabular}

NLR: neutrophil to lymphocyte ratio; SD: standard deviation; F/M: female/male; FACIT fatigue scale: the Functional Assessment of Chronic IIIness Therapy fatigue scale.

Table 2. The Comparisons of Demographic and Clinical Features and the Results of Questionnaires Between Episodic Migraineurs and Chronic Migraineurs

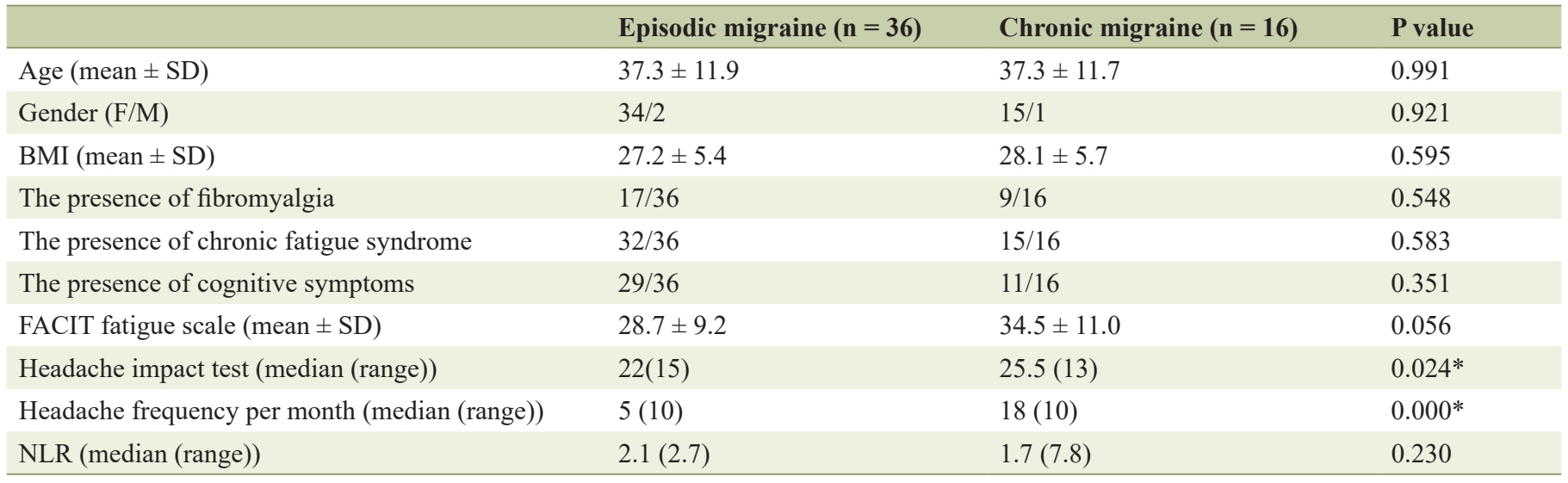

${ }^{*} \mathrm{P}<0.05$. NLR: neutrophil to lymphocyte ratio; SD: standard deviation; F/M: female/male; BMI: body mass index; FACIT fatigue scale: the Functional Assessment of Chronic Illness Therapy fatigue scale.

of migraines, including severity, comorbidity, or any accompanying symptom. Correlation analyses of the NLR also did not yield a significant result.

Neurogenic inflammation around the dural trigeminal af- ferents, plasma protein extravasation, and vasodilation play important roles in the generation of migraine attacks. Therefore, several studies have been conducted on the inflammatory aspects of migraine, and the possible associations between

Table 3. The Possible Utility of NLR in Discriminating the Migraineurs According to the Presence of Fibromyalgia, Chronic Fatigue Syndrome, Tinnitus and Cognitive Symptoms, Respectively

\begin{tabular}{ll}
\hline & The results of the comparisons of NLR between the patient groups (P values) \\
\hline Episodic and chronic migraine & 0.230 \\
FM (+) and FM (-) groups & 0.647 \\
CFS (+) and CFS (-) groups & 0.331 \\
Tinnitus (+) and tinnitus (-) groups & 0.721 \\
Cognitive symptoms (+) and (-) groups & 0.143 \\
\hline
\end{tabular}

NLR: neutrophil to lymphocyte ratio; FM: fibromyalgia: CFS: chronic fatigue syndrome. 
Table 4. The Results of the Correlation Analyses

\begin{tabular}{lll}
\hline The correlation analyses & The correlation coefficient (r) & Pvalue \\
\hline NLR/FACIT fatigue scale & -0.226 & 0.108 \\
NLR/HIT-6 & -0.090 & 0.526 \\
FACIT fatigue scale/HIT-6 & 0.322 & $0.020^{*}$ \\
FACIT fatigue scale/headache frequency per month & 0.497 & $0.000^{*}$ \\
BMI/FACIT fatigue scale score & -0.007 & 0.962 \\
BMI/headache frequency per month & -0.072 & 0.614 \\
\hline
\end{tabular}

${ }^{*} \mathrm{P}<0.05$. NLR: neutrophil to lymphocyte ratio; FACIT fatigue scale: the Functional Assessment of Chronic Illness Therapy fatigue scale; HIT-6: headache impact test; BMI: body mass index.

various inflammatory biomarkers and migraines have been investigated by several researchers $[3,19,20]$. Many researchers have investigated the relationships between the features of headaches and inflammation using hematologic parameters [3, $6,10,13,21-23]$. The main goal of these studies is to evaluate the inflammatory processes using hematological markers and to investigate the association of these markers with clinical parameters of headaches, and their utility in discriminating headache syndromes from other diseases [10, 24]. A critical study by Ceylan et al investigating inflammatory markers in serum in migraine patients found higher levels of fibrinogen and pentraxin-3 (PTX-3) in migraine patients suffering acute attacks as compared to those in healthy control patients. However, the same study found no significant difference in $\mathrm{C}$-reactive protein (CRP) levels between the patient groups within the attack and interictal period [24]. Remarkably, they found higher serum levels of fibrinogen and PTX-3 in migraine attack patients compared with patients during the interictal period [24]. Among the inflammatory markers used in the clinical practice, WBC count is a crucial one used also widely in scientific researches. Remarkably, an increased "NLR" has been emphasized to reflect inflammation and inflammation-related pathologies [4]. NLR is an easily measured, reproducible, and inexpensive marker, and it is a parameter to be used also for subclinical inflammation [25]. Therefore, its utility on the clinical grounds including its diagnostic significance, prognostic role, and its possible contribution regarding the underlying pathophysiology has constituted an attractive topic of research interest $[7,8,10]$. The prognostic or diagnostic role of NLR has been investigated rather in a limited number of studies focusing on neurological disorders, which included patients with acute stroke, subarachnoid hemorrhage, restless leg syndrome, multiple sclerosis, and headaches $[6-8,10,13,26,27]$. To the best of our knowledge, there are only three previous reports evaluating the significance of the NLR for the evaluation of patients with migraine $[8,10$, 13]. In the study by Karabulut et al on 92 patients with migraines during the attack period, the value of NLR was found to be higher in the patients, compared to the controls [10]. However, in this study, migraineurs during the interictal period were not investigated. Eryigit et al also did not find any statistically significant difference in the NLR between migraine and other patients with primary headache syndromes [8]. Nevertheless, this study was in a retrospective design and no data were given regarding if the migraineurs were during the attack period or interictal period. The other study was designed in a prospective manner, which included 260 patients with migraines and 260 healthy people [13]. In conclusion, the authors did not find a difference in the NLR between migraineurs and healthy control group. However, the major limitation of this study was also that no data regarding the period of patients (attack or interictal period) at the time of obtaining blood samples was noted. Ergo, we think that the negative results of our study in light of the above-mentioned studies may give crucial perspectives for further deliberations. These results may suggest that although the NLR may be a marker to distinguish patients with migraine attacks from healthy controls and other headache syndromes during the interictal period, it may not have significance in the differential diagnostic processes of migraine or distinguishing patients from healthy individuals. Besides, we did not find an association between the NLR and the clinical parameters including severity, other comorbidities or any other symptoms. The comprehensive assessments of the clinical characteristics of migraine as in our study were not made in the above-mentioned previous reports.

Based on these conclusions, we suggest that the inflammatory process which we evaluated measuring the NLR may not be a noteworthy factor in the diagnosis of migraine or prediction of any migraine characteristics or subgroups. However, its association with migraine attacks which has been shown in the previous report [10], may potentially be a useful point in the differentiation of migraine attacks from other types of coincidental causes of headaches in migraineurs, such as radicular headache, hypertensive headache or somatic symptoms. Remarkably, although migraine is a common and a well-known disease, the differentiation of migraine attacks from other causes such as tension-type headaches, intracranial tumor or aneurysm may be strictly challenging in some atypical scenarios. Therefore, the potential utility of the NLR in the differential diagnosis of migraine attacks may still be a critical issue albeit the negative results of our study. In our study, we have not included migraineurs during attack periods, which avoids making conclusions in this regard. However, our hypothesis may constitute a critical topic of interest to be investigated in future related studies.

Another result of this study was that we found a significant correlation between the FACIT fatigue scale score and the parameters of migraine severity. The increased risk of CFS in migraineurs has been shown in previous large-scale studies 
$[28,29]$. Increasing evidence suggests that both disorders may share common pathophysiological mechanisms such as mitochondrial dysfunction and central sensitization [28]. Although including a low number of patients, the significant correlations we found between the score of the FACIT fatigue scale and the parameters of migraine severity also support the previous study results. We think that the investigation of this association in future studies evaluating the clinical features of migraineurs in detail may also provide substantial contributions regarding the underlying pathophysiological mechanisms.

The limitation of our study was that we included a small number of patients, and we did not include healthy control individuals. However, a comprehensive assessment of migraine characteristics has been performed that was not evaluated in the previous studies investigating the significance of the NLR in migraine, which makes our study results certainly substantial. Second, we have not included a group of migraineurs during a migraine attack. Including the subgroup of patients during migraine attack may enable to evaluate the possible utility of the NLR in discriminating patients with an attack from others and the pathophysiological role of inflammation in a migraine attack. Another limitation may be that we did not comparatively investigate the NLR in migraineurs with aura and without aura. The data regarding the presence of aura had not been evaluated in some patients for whom we did not include this data in the study which surely needs to be investigated in future studies.

In conclusion, we suggest that the NLR cannot be a specific marker to be used in the differential diagnosis or prediction of any features of migraines during the interictal period. However, in light of the previous reports, the utility of this value in the differentiating of migraine attacks from other causes of headache attacks can be investigated in future related studies.

\section{Acknowledgments}

None to declare.

\section{Financial Disclosure}

The authors declared that this study has received no financial support.

\section{Informed Consent}

Informed consents have been obtained from the participants.

\section{Conflict of Interest}

The authors have no conflict of interest to declare.

\section{Author Contributions}

Study concept: HO; design: HO; supervision: HO; materials:
$\mathrm{HO}$ and MMD; data collection and/or processing: $\mathrm{HO}$ and MMD; analysis and/or interpretation: $\mathrm{HO}$; literature search: $\mathrm{HO}$; writing manuscript: $\mathrm{HO}$ and MMD; critical review: HO.

\section{Data Availability}

The data supporting the findings of this study are available from the corresponding author upon reasonable request.

\section{References}

1. Harriott AM, Takizawa T, Chung DY, Chen SP. Spreading depression as a preclinical model of migraine. J Headache Pain. 2019;20(1):45.

2. Ramachandran R. Neurogenic inflammation and its role in migraine. Semin Immunopathol. 2018;40(3):301-314.

3. Vanmolkot FH, de Hoon JN. Increased C-reactive protein in young adult patients with migraine. Cephalalgia. 2007;27(7):843-846.

4. Rudiger A, Burckhardt OA, Harpes P, Muller SA, Follath F. The relative lymphocyte count on hospital admission is a risk factor for long-term mortality in patients with acute heart failure. Am J Emerg Med. 2006;24(4):451-454.

5. Wada H, Dohi T, Miyauchi K, Shitara J, Endo H, Doi S, Konishi H, et al. Pre-procedural neutrophil-to-lymphocyte ratio and long-term cardiac outcomes after percutaneous coronary intervention for stable coronary artery disease. Atherosclerosis. 2017;265:35-40.

6. Kotfis K, Bott-Olejnik M, Szylinska A, Rotter I. Could Neutrophil-to-Lymphocyte Ratio (NLR) serve as a potential marker for delirium prediction in patients with acute ischemic stroke? A Prospective Observational Study. J Clin Med. 2019;8(7):1075.

7. Hemond CC, Glanz BI, Bakshi R, Chitnis T, Healy BC. The neutrophil-to-lymphocyte and monocyte-to-lymphocyte ratios are independently associated with neurological disability and brain atrophy in multiple sclerosis. BMC Neurol. 2019;19(1):23.

8. Eryigit U, Altunayoglu Cakmak V, Sahin A, Tatli O, Pasli S, Gazioglu G, Karaca Y. The diagnostic value of the neutrophil-lymphocyte ratio in distinguishing between subarachnoid hemorrhage and migraine. Am J Emerg Med. 2017;35(9):1276-1280.

9. Bressi E, Mangiacapra F, Ricottini E, Cavallari I, Colaiori I, Di Gioia G, Creta A, et al. Impact of neutrophil-to-lymphocyte ratio and platelet-to-lymphocyte ratio on 5-year clinical outcomes of patients with stable coronary artery disease undergoing elective percutaneous coronary intervention. J Cardiovasc Transl Res. 2018;11(6):517-523.

10. Karabulut KU, Egercioglu TU, Uyar M, Ucar Y. The change of neutrophils/lymphocytes ratio in migraine attacks: A case-controlled study. Ann Med Surg (Lond). 2016;10:52-56.

11. Faria SS, Fernandes PC, Jr., Silva MJ, Lima VC, Fontes W, Freitas-Junior R, Eterovic AK, et al. The neutrophilto-lymphocyte ratio: a narrative review. Ecancermedicalscience. 2016;10:702. 
12. Nagy GR, Kemeny L, Bata-Csorgo Z. Neutrophil-tolymphocyte ratio: a biomarker for predicting systemic involvement in adult IgA vasculitis patients. J Eur Acad Dermatol Venereol. 2017;31(6):1033-1037.

13. Aynur Yilmaz Avci OA. Migraine and peripheral inflammation. Acta Medica Alanya. 2017;1:127-134.

14. Headache Classification Committee of the International Headache S. The International Classification of Headache Disorders, 3rd edition (beta version). Cephalalgia. 2013;33:629-808.

15. Arnold LM, Bennett RM, Crofford LJ, Dean LE, Clauw DJ, Goldenberg DL, Fitzcharles MA, et al. AAPT diagnostic criteria for fibromyalgia. J Pain. 2019;20(6):611628.

16. Chronic fatigue syndrome/myalgic encephalomyelitis (or encephalopathy). Diagnosis and management of chronic fatigue syndrome/myalgic encephalomyelitis (or encephalopathy) in adults and children. London, 2007.

17. Kosinski M, Bayliss MS, Bjorner JB, Ware JE, Jr., Garber WH, Batenhorst A, Cady R, et al. A six-item short-form survey for measuring headache impact: the HIT-6. Qual Life Res. 2003;12(8):963-974.

18. Chandran V, Bhella S, Schentag C, Gladman DD. Functional assessment of chronic illness therapy-fatigue scale is valid in patients with psoriatic arthritis. Ann Rheum Dis. 2007;66(7):936-939.

19. Ridker PM. Moving beyond JUPITER: will inhibiting inflammation reduce vascular event rates? Curr Atheroscler Rep. 2013;15(1):295.

20. Williamson DJ, Hargreaves RJ. Neurogenic inflammation in the context of migraine. Microsc Res Tech. 2001;53(3):167-178.
21. Yazar HO, Yazar T, Aygun A, Kaygisiz S, Kirbas D. Evaluation of simple inflammatory blood parameters in patients with migraine. Ir J Med Sci. 2019.

22. Martami F, Razeghi Jahromi S, Togha M, Ghorbani Z, Seifishahpar M, Saidpour A. The serum level of inflammatory markers in chronic and episodic migraine: a casecontrol study. Neurol Sci. 2018;39(10):1741-1749.

23. Lippi G, Mattiuzzi C, Cervellin G. C-reactive protein and migraine. Facts or speculations? Clin Chem Lab Med. 2014;52(9):1265-1272.

24. Ceylan M, Bayraktutan OF, Becel S, Atis O, Yalcin A, Kotan D. Serum levels of pentraxin-3 and other inflammatory biomarkers in migraine: Association with migraine characteristics. Cephalalgia. 2016;36(6):518-525.

25. McMillan DC. Systemic inflammation, nutritional status and survival in patients with cancer. Curr Opin Clin Nutr Metab Care. 2009;12(3):223-226.

26. Luo Y, Xia LX, Li ZL, Pi DF, Tan XP, Tu Q. Early neutrophil-to-lymphocyte ratio is a prognostic marker in acute minor stroke or transient ischemic attack. Acta Neurol Belg. 2020.

27. Ali Zeynal Abidin Tak YS. Evaluation of inflammation with neutrophil-to-lymphocyte ratio and platelet-to-lymphocyte ratio in restless legs syndrome. Turkish Journal of Neurology. 2018;24:259-263.

28. Lau CI, Lin CC, Chen WH, Wang HC, Kao CH. Increased risk of chronic fatigue syndrome in patients with migraine: A retrospective cohort study. J Psychosom Res. 2015;79(6):514-518.

29. Peres MF, Zukerman E, Young WB, Silberstein SD. Fatigue in chronic migraine patients. Cephalalgia. 2002;22(9):720-724. 